Journal of trends in Computer Science and Smart technology (TCSST) (2020)

Vol.02/ No. 01

Pages: 56- 68

https://www.irojournals.com/tcsst/

DOI: https://doi.org/10.36548/jtcsst.2020.1.006

\title{
Interoperability Improvement in Internet of Things Using Fog Assisted Semantic Frame Work
}

\author{
Dr. Joy Iong Zong Chen \\ Professor, Department of Electrical Engineering \\ Da-Yeh University \\ Taiwan \\ Email id: jchen@mail.dyu.edu.tw \\ Dr. S. Smys \\ Professor, \\ Department of Computer Science Engineering \\ RVS technical Campus \\ Coimbatore, India \\ Email:smys375@gmail.com
}

\begin{abstract}
Cloud computing is equipped with the numerous of advantageous features to support software and utilities on the Internet of Things (IoT). Cloud-based technology is widely used when offering support for heterogeneous applications integrating specific IoT that follows various semantics. Attaching additional information to raw data sensed with the help of ontology is accomplished in semantic model. The longer distance between the cloud and IoT applications, however, is a bottleneck for vital IoT software. So the paper puts forth a semantic frame work assisted by the fog to enhance the interoperability in the internet of things. The structure put forth moves some of the cloud's commonly used semantic resources sensor networks edge and also offers an effective offloading technique between fog - fog and fog - cloud devices to diminish total computation time of the task and the energy consumed by the nodes in the fog. The proposed method further follows an efficient mapping technique to transform the data's sensed into a RDF-format such that it is compatible for processing. The proposed model is evaluated on the basis of delay in the service provision, the energy consumed, and the total cost of the system and further the results obtained are compared with the relevant cloud based computing models, to reveal the proficiency of the proposed.
\end{abstract}

Keywords: Cloud Computing, Semantic Frame Work, Fog Computing, Internet of Things, Interoperability Improvement

\section{Introduction}

The latest technological progress and the advancements in the communication has made possible the internet of things that is empowered with the multitudes of sensors, and various internet protocols. The development of the internet of things has changed the life style of humans by enabling the things around us

ISSN: 2582-4104 
Journal of trends in Computer Science and Smart technology (TCSST) (2020)

Vol.02/ No. 01

Pages: 56- 68

https://www.irojournals.com/tcsst/

DOI: https://doi.org/10.36548/jtcsst.2020.1.006

to stay connected using the internet. The communication between the devices takes place without any human intervention by involving diverse group of devices (hard ware and software) and the operating system that are heterogeneous. The heterogeneous devices transmit information using any one of the various communication protocols ( CoAP- constrained application protocol), MQTT, - message queuing protocol, XMPP- extensible messaging and presence protocol, and the AMPQ- advanced message queuing protocol) available utilizing the technologies such as the Bluetooth, zigbee, RFID, Wi-Fi, Wi-max etc. to extend communication. Certainly these technologies as well as the protocols ensure a far better seamless communication that could not be afforded by the conventional computing systems. In the future the devices in the internet of things are anticipated to operate with improved interoperable capability with the technologies and the protocols and the devices that are of different capabilities to extend its accessibility to a broad range of applications.

Every data packets that are produced from the various tangible things connected to the internet of things would be portrayed with syntax, formats and the types that are different and its semantics also differs, for instance the suppose a two persons belonging to two different countries happens to communicate using the social network, using their own mother tongue to transfer information's. Such services would be made possible by the interoperability that offers a common services for the applications by assimilating the protocols, semantics the technologies used in communication as well the devices that are heterogeneous. The semantic interoperability in the IOT environs that is heterogeneous, the ontology is utilized to equip the raw data that are sensed with the semantic annotations. These functionalities are more commonly instigated in cloud due to its processing capabilities, but experiences a delay in the services as the as the speed in responding for the requisition of the users does not match its processing speed. Although these centralized frameworks, fulfill the desire of numerous of applications, their consumption rate of the time, energy and the bandwidth are very high. These basic restrictions that prevail in the connectivity between the cloud and the devices at the user end make them unsuitable for the IoT based applications such as the real time traffic management, health support and fraudulent detection etc. that require immediate responses.

A real time queries have time constraints and demands responses before the deadline of the query is reached. The evolution of the fog computing enabled the execution of the real time applications with the time limitations at the user end with the assistance of the edge devices. It further provided other benefits to the users such as the minimized latency due to the execution taking place in local processors. Minimized energy consumption and enhanced bandwidth utilization as the repeated as well as the majority of associated information's are not taken to the cloud for processing and computed close to the user devices.

ISSN: 2582-4104 
Journal of trends in Computer Science and Smart technology (TCSST) (2020)

Vol.02/ No. 01

Pages: 56- 68

https://www.irojournals.com/tcsst/

DOI: https://doi.org/10.36548/jtcsst.2020.1.006

Although variety of Fog founded architecture are devised in the existing works to carry out effectively the issues that are associated with the heterogeneity of the devices the strategies that accomplish the semantic frame work enhancing the Iot interoperability were not implemented into the devices in the FOG layer.

So to achieve this the semantic frame work that was centralized in the FOG was developed, the devised model was improved further with the better offloading technique to diminish total computation time of the task and the energy consumed by the nodes in the fog. The proposed method further follows an efficient mapping technique to transform the data's sensed into a RDF-format such that it is compatible for processing.

The paper providing an effective semantic model that is centralized in fog to deliver the meanings for the data sensed is formed with the related works in the section 2 that depicts the various architecture of the FOG and how semantic for the data enables to have an enhanced interoperability. The section 3 present $\mathrm{s}$ the objective functions of the proposed offloading and mapping, the section 4 elaborates the proposed semantic frame work and the techniques used in offloading and mapping. The evaluation of the system performance in carried out in section 5, followed by the conclusion in the section 6 .

\section{Related works}

Kumar, T. Senthil et al [1] has put forth the "Efficient resource allocation and QOS enhancements of IoT with FOG network." Mugunthan, S. R. et al [2] has proposed "Security and Privacy Preserving of Sensor Data Localization Based on Internet of Things." Bestak, et al [3] has put forth the "Big Data Analytics for Smart Cloud-Fog Based Applications." Pandian, M. Durai et al [4] has proffered the "Enhanced Network Performance and Mobility Management of Iot Multi Networks." Bashar, Abul. et al [5] presents the "Secure And Cost Efficient Implementation Of The Mobile Computing Using Offloading Technique." Smys, S., et al [6] has disclosed an "Internet of Things and Big Data Analytics for Health Care with Cloud Computing."

Mouradian, et al [7] elaborates the "A comprehensive survey on fog computing: State-of-the-art and research challenges." Smys, S., and Joy Iong-Zong Chen et al [8] elaborates the "Special Section on Innovative Engineering Solutions for Future Health Care Informatics." Elkhodr, et al [9] has presented a "The internet of things: new interoperability, management and security challenges." Lee, et al [10] has proposed a "A gateway based fog computing architecture for wireless sensors and actuator networks." Kovacs, et al [11] has proffered a "Standards-based worldwide semantic interoperability for IoT."

ISSN: 2582-4104 
Journal of trends in Computer Science and Smart technology (TCSST) (2020)

Vol.02/ No. 01

Pages: 56- 68

https://www.irojournals.com/tcsst/

DOI: https://doi.org/10.36548/jtcsst.2020.1.006

Agarwal, et al [12] has put forward "An efficient architecture and algorithm for resource provisioning in fog computing." Shi, Heng, et al [13] has put forward " coap to link mobile device clouds with fog computing by Combining mobile and fog computing" Al-Fuqaha et al [14], has proposed a " A survey on enabling technologies, protocols, and applications." Giang, et al [15] proposed a "Developing iot applications in the fog with a distributed dataflow approach." Hong, et al [16] put forward a "Mobile fog proposing a programming model for large-scale applications on the internet of things." Bonomi, et al [17] elaborates the part of the Fog computing in the Internet of things paradigm.

\section{Objective functions}

The objective functions that are to be optimized using the proposed method is list below

The first objective is the delay incurred in the services in the cloud is optimized in the proposed model as major of the requests are executed in the local device that closer to the user end. The equation 1 is framed such in this regard.

Delay in fog $=\sum$ (time taken to transmit and receive) $+\sum$ time of semantic modelling + $\sum$ Distance

The next objective is the energy utilization that depends on the computation and the transmission as well as the reception of the request and its responses respectively. So the energy utilized is calculated as shown in the equation 2

Energy $=$ Senergy consumed per bit transmission and reception $*$ delay incurred

Further the network utilization that depends on the amount of bandwidth used is estimated. As the proposed model segregates the important data from the repeated data and stops the transmission of the irrelevant data packets alone to the fog node capable of processing the requests, the network usage is reduce by 3

$$
\text { Network usage }=\left[\frac{\text { NUmber of data packets }}{\text { Fog bandwidth }}\right] * 100
$$

ISSN: 2582-4104 
Journal of trends in Computer Science and Smart technology (TCSST) (2020)

Vol.02/ No. 01

Pages: 56- 68

https://www.irojournals.com/tcsst/

DOI: https://doi.org/10.36548/jtcsst.2020.1.006

And finally the average cost is much minimized compared to the computing that takes place in the cloud. The average cost for computing in the fog is given as in equation 4

$$
\text { Total cost }=\text { Computing_cost }+ \text { storage_cost }+ \text { communication_cost }
$$

\section{Proposed Work}

To extract the data with the highest value, from the enormous amount information's that are sensed devices associated with the internet of things, computing the information's becomes indispensable causing the world to be smarter by gaining valuable information's from the original form of data that are sensed, for this purpose the "semantic annotations" that assign meanings to the data gathered and allows make accurate decisions on the service requisition. The diagram below in the figure. 1 shows the various operations involved in a semantic frame work to assign the accurate meanings to the data sensed.

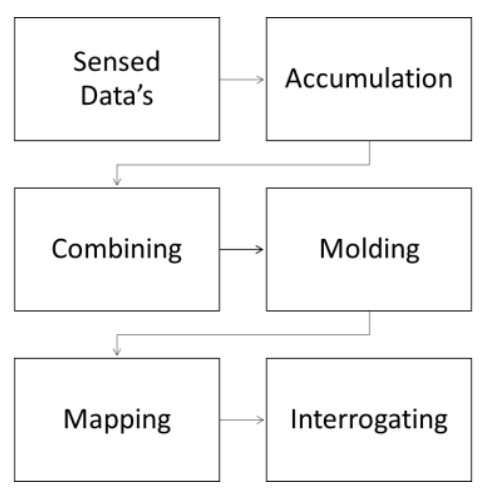

Figure.1 Essential Parts of Semantic Frame work

This process was traditionally facilitated by the cloud, with the data that were accumulated being forwarded to the cloud for filtering, molding that is modeling, mapping and annotating its meaning as well as reasoning to have an enhanced computing. But this computation handled in cloud consumed more time as well as energy and bandwidth resulting unsuitable for the requisition with the time constraints. So to minimize the time of computation along with the energy, latency, bandwidth utilization and cost. The semantic framework from the cloud is shifted to the cloud. A hierarchical multitier Fog network is developed with

ISSN: 2582-4104 
Journal of trends in Computer Science and Smart technology (TCSST) (2020)

Vol.02/ No. 01

Pages: 56- 68

https://www.irojournals.com/tcsst/

DOI: https://doi.org/10.36548/jtcsst.2020.1.006

two layers, to perform the semantic annotations and the mapped data are offered to the cloud for processing if it is not time constrained and requires high computational capabilities. By this heavy flow of user requisitions to the cloud is stopped and the much of the energy consumption and the time are saved. The figure. 2 is the fog based semantic framework for the IOT services.

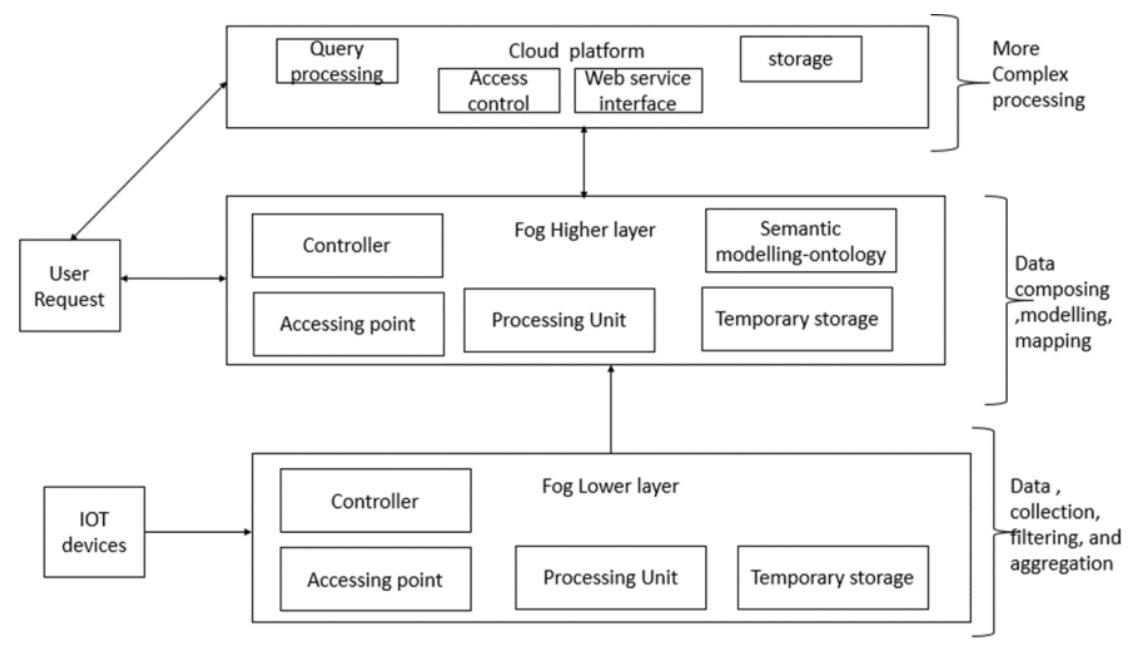

Figure. 2 Proposed Fog Structure

As shown in the figure. 2 the sensors or the IOT devices convey the information over the low layer of the FOG, this layer filters the appropriate and the repeated data, accumulates the data filtered with the high quality and offloads it to the next- higher layer in the FOG, utilizing the Single-owl -file ontology that is educated about and interconnected with the radio frequency identification, sensors and actuators, the data in are attached with the meaningful annotations, it is light weight ontology which is integrated into the proposed architecture using the middle Ware that is compatible with it, this middle ware converts the data that is composed and modelled using the meaningful annotations into a format that is more suitable for computing, the format followed here is the RDF format the steps shown in the figure. 3 is describes the process of mapping the data into a RDF format.

ISSN: 2582-4104 
Journal of trends in Computer Science and Smart technology (TCSST) (2020)

Vol.02/ No. 01

Pages: 56- 68

https://www.irojournals.com/tcsst/

DOI: https://doi.org/10.36548/jtcsst.2020.1.006

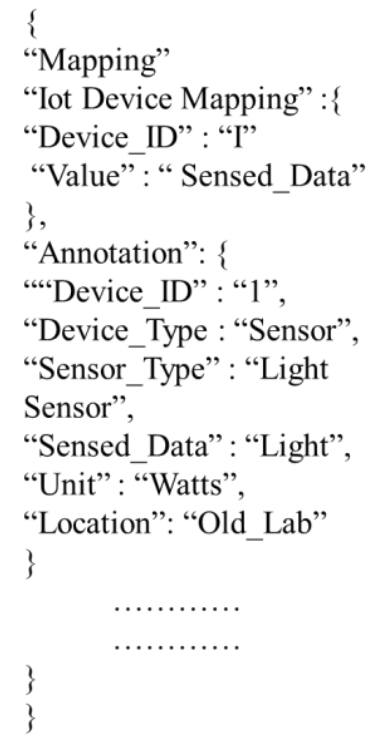

Figure.3.Mapping of Data

The semantic annotation is attached to the data, by extricating the conceptions as well as properties from the ontology and linking it to the data that are gathered from the devices of the internet of things, the proposed method utilizes the JSON for the mapping of the Data as shown in fig.3, this a "light weight java script object notation" providing the association between the strategy as well as the ontology. These mapped data are processed in the FOG based on their computational complexities and this decided by the controller located in the FOG nodes.

Based on the time constraints on the requests, the computing capacity of the nodes in the FOG and the average length of the requests queued, the controller in the FOG layer decides the whether the off-loading has to take place within the fog nodes are to the cloud, usually the request with latency tolerance and that which demands a storage for long term are always directed to the cloud. The steps below describes the offloading among the FOG nodes and the way the nodes are selected.

ISSN: 2582-4104 
Journal of trends in Computer Science and Smart technology (TCSST) (2020)

Vol.02/ No. 01

Pages: 56- 68

https://www.irojournals.com/tcsst/

DOI: https://doi.org/10.36548/jtcsst.2020.1.006

Step. 1 : Initialize the Fog nodes

Step 2 : Check for uploaded Request

Step 3 : Select the Offloading nodes.

Step 4 : Estimate the delay tolerance, energy consumption and the memory requirement for the requests.

Step 5 : Estimate the processing capacity of the Fog Nodes

Step 6 : Estimate the distance of the Fog Nodes to the request location

Step 7 : If the distance, delay tolerance, the energy consumption and the memory requirement are satisfied Found satisfying.

Step 8 : The Data are processed in the FOG nodes.

Step 9: Else directed to the Cloud

Thus the proposed model enables to perform a semantic modelling in the fog node itself and further process the data in the fog node itself based on its computational requirements of the requests and the evaluation of the proposed model using the network simulator -2 described in the next section shows the capability of the proposed method on the basis of the energy used up, delay in the services and the average e cost as well sas the network utilization.

\section{Performance Evaluation}

The proposed strategy was evaluated using the network simulator -2 on the basis of delay incurred in the service, the energy utilized, the network utilization and the average cost, the results obtained where further compared with the conventional centralized cloud where the semantic model is executed in the cloud.

ISSN: 2582-4104 
Journal of trends in Computer Science and Smart technology (TCSST) (2020)

Vol.02/ No. 01

Pages: 56- 68

https://www.irojournals.com/tcsst/

DOI: https://doi.org/10.36548/jtcsst.2020.1.006

\begin{tabular}{|l|l|}
\hline \multicolumn{1}{|c|}{ Parameter } & \multicolumn{1}{c|}{ Values } \\
\hline simulation duration & $1000 * 1000 \mathrm{~ms}$ \\
\hline Number of User Request & 100 \\
\hline Energy per Node & 2 joules \\
\hline up-link bandwidth & $50-100 \mathrm{Mbps}$ \\
\hline down-link bandwidth & $10-50 \mathrm{Mbps}$ \\
\hline RAM & $1000 \mathrm{Mb}$ \\
\hline Number of Fog Nodes & 10 \\
\hline
\end{tabular}

Table.1 Simulation Parameter

The table .1 is the particulars of the parameters utilized in the simulating the proposed model.

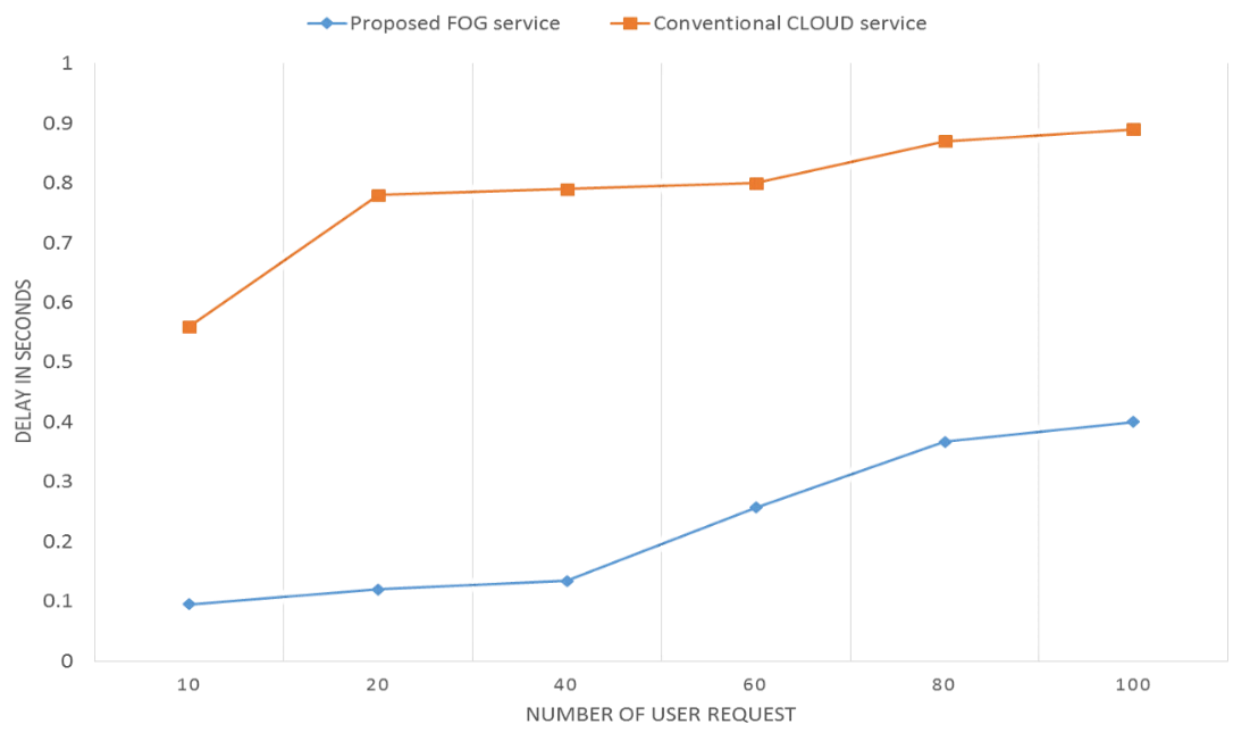

Figure. 4 Delay in the Service

ISSN: 2582-4104 
Journal of trends in Computer Science and Smart technology (TCSST) (2020)

Vol.02/ No. 01

Pages: 56- 68

https://www.irojournals.com/tcsst/

DOI: https://doi.org/10.36548/jtcsst.2020.1.006

The results in fig. 4 shows the delay observed in the while executing the user request in the FOG and the Cloud, the delay incurred shows that the proposed method has faster response compared to the cloud as the execution takes place the local device that is closer to the user end.

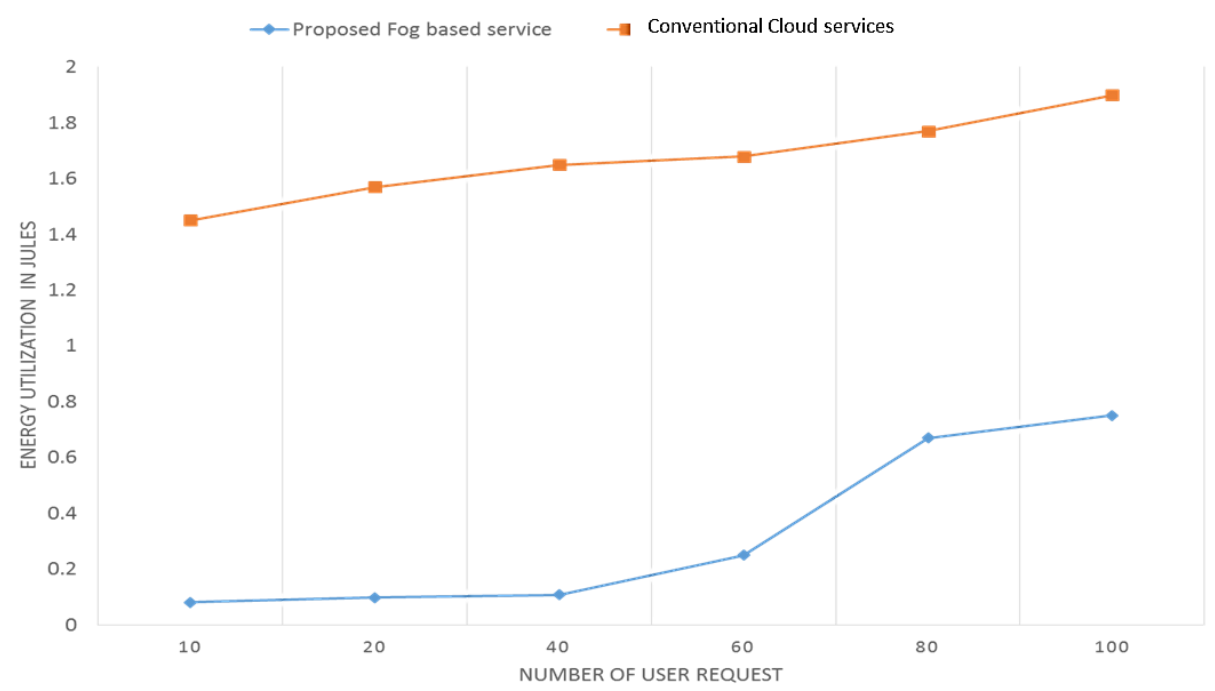

Figure. 5 Energy Utilization

The fig. 5 shows the results observed on the energy utilized in the on computing and transmitting the requests to the within the FOG and the Cloud. The Results shows that the proposed method has minimized the majority of its energy utilization as the execution takes place in a device very close to the user devices.

ISSN: 2582-4104 
Journal of trends in Computer Science and Smart technology (TCSST) (2020)

Vol.02/ No. 01

Pages: 56- 68

https://www.irojournals.com/tcsst/

DOI: https://doi.org/10.36548/jtcsst.2020.1.006

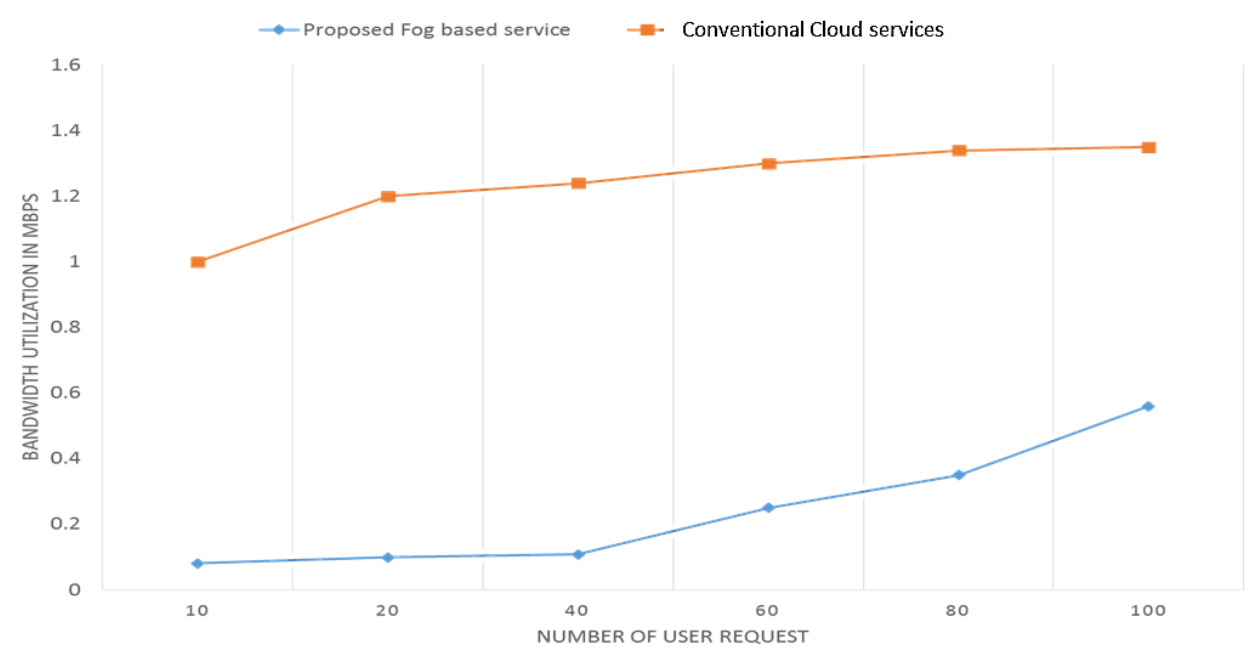

Figure. 6 Bandwidth Utilization

The Fig. 6 provides the Bandwidth Utilization of the proposed model, the bandwidth utilization of the proposed model is much reduced compared to the conventional cloud as most of the irrelevant data are reduced by the process of filtering. The table. 2 is the average cost observed on the proposed and the conventional model for different number of user request.

\begin{tabular}{|c|c|c|}
\hline $\begin{array}{l}\text { No. of User } \\
\text { Request }\end{array}$ & $\begin{array}{l}\text { Proposed Fog } \\
\text { Service (\$) }\end{array}$ & $\begin{array}{l}\text { Conventional } \\
\text { Cloud Services (\$) }\end{array}$ \\
\hline 10 & 15 & 45 \\
\hline 20 & 20 & 56 \\
\hline 30 & 24 & 57 \\
\hline 40 & 27 & 59 \\
\hline 50 & 30 & 60 \\
\hline 60 & 34 & 63 \\
\hline 70 & 37 & 65 \\
\hline 80 & 40 & 75 \\
\hline 90 & 45 & 80 \\
\hline 100 & 49 & 95 \\
\hline
\end{tabular}

Table. 2 Average Cost

ISSN: 2582-4104 
Journal of trends in Computer Science and Smart technology (TCSST) (2020)

Vol.02/ No. 01

Pages: 56- 68

https://www.irojournals.com/tcsst/

DOI: https://doi.org/10.36548/jtcsst.2020.1.006

\section{Conclusion}

The paper has devised a Fog centered semantic model for enhancing the interoperability of the IOT devices with a much reduced energy utilization, delay in the service, bandwidth utilization and cost, by devising a proper offloading procedure that prevents the major of the requests from being executed in the cloud. The semantic modeling is totally taken care by the FOG in the proposed model this further reduces the amount of irrelevant data being offloaded thus minimizing the major consumption of the bandwidth minimizing the network usage, and the total cost, the single-owl-file ontology used in the paper and the RDF mapping done to add meaningful annotations to the data further enhances the interoperability in the IOT devices. The proposed method was simulated using the network simulator- 2 and its performance was evaluated on the basis of the latency, energy usage, network utilization and the cost of processing. This results attained for the proposed frame work were much better compared to the capability of the conventional cloud in terms of latency, energy usage, network utilization and the cost of processing.

\section{References}

[1] Kumar, T. Senthil. "Efficient resource allocation and QOS enhancements of IoT with FOG network." J ISMAC 1 (2019): 101-110.

[2] Mugunthan, S. R. "Security and Privacy Preserving Of Sensor Data Localization Based On Internet Of Things." Journal of ISMAC 1, no. 02 (2019): 81-91.

[3] Bestak, Robert, and S. Smys. "Big Data Analytics for Smart Cloud-Fog Based Applications." Journal of trends in Computer Science and Smart technology (TCSST) 1, no. 02 (2019): 7483.

[4] Pandian, M. Durai. "Enhanced Network Performance and Mobility Management Of Iot Multi Networks." Journal of trends in Computer Science and Smart technology (TCSST) 1, no. 02 (2019): 95-105.

[5] Bashar, Abul. "Secure And Cost Efficient Implementation Of The Mobile Computing Using Offloading Technique." Journal of Information Technology 1, no. 01 (2019): 48-57.

[6] Smys, S., and Jennifer S. Raj. "Internet of Things and Big Data Analytics for Health Care with Cloud Computing." Journal of Information Technology 1, no. 01 (2019): 9-18.

[7] Mouradian, Carla, Diala Naboulsi, Sami Yangui, Roch H. Glitho, Monique J. Morrow, and Paul A. Polakos. "A comprehensive survey on fog computing: State-of-the-art and research challenges." IEEE Communications Surveys \& Tutorials 20, no. 1 (2017): 416-464.

ISSN: 2582-4104 
Journal of trends in Computer Science and Smart technology (TCSST) (2020)

Vol.02/ No. 01

Pages: 56- 68

https://www.irojournals.com/tcsst/

DOI: https://doi.org/10.36548/jtcsst.2020.1.006

[8] Smys, S., and Joy Iong-Zong Chen. "Special Section on Innovative Engineering Solutions for Future Health Care Informatics." Journal of Medical Imaging and Health Informatics 6, no. 7 (2016): 1570-1571.

[9] Elkhodr, Mahmoud, Seyed Shahrestani, and Hon Cheung. "The internet of things: new interoperability, management and security challenges." arXiv preprint arXiv:1604.04824 (2016).

[10] Lee, Wangbong, Kidong Nam, Hak-Gyun Roh, and Sang-Ha Kim. "A gateway based fog computing architecture for wireless sensors and actuator networks." In 2016 18th International Conference on Advanced Communication Technology (ICACT), pp. 210-213. IEEE, 2016.

[11] Kovacs, Erno, Martin Bauer, Jaeho Kim, Jaeseok Yun, Franck Le Gall, and Mengxuan Zhao. "Standards-based worldwide semantic interoperability for IoT." IEEE Communications Magazine 54, no. 12 (2016): 40-46.

[12] Agarwal, Swati, Shashank Yadav, and Arun Kumar Yadav. "An efficient architecture and algorithm for resource provisioning in fog computing." International Journal of Information Engineering and Electronic Business 8, no. 1 (2016): 48.

[13] Shi, Heng, Nan Chen, and Ralph Deters. "Combining mobile and fog computing: Using coap to link mobile device clouds with fog computing." In 2015 IEEE International Conference on Data Science and Data Intensive Systems, pp. 564-571. IEEE, 2015.

[14] Al-Fuqaha, Ala, Mohsen Guizani, Mehdi Mohammadi, Mohammed Aledhari, and Moussa Ayyash. "Internet of things: A survey on enabling technologies, protocols, and applications." IEEE communications surveys \& tutorials 17, no. 4 (2015): 2347-2376.

[15] Giang, Nam Ky, Michael Blackstock, Rodger Lea, and Victor CM Leung. "Developing iot applications in the fog: A distributed dataflow approach." In 2015 th International Conference on the Internet of Things (IOT), pp. 155-162. IEEE, 2015.

[16] Giang, Nam Ky, Michael Blackstock, Rodger Lea, and Victor CM Leung. "Developing iot applications in the fog: A distributed dataflow approach." In 2015 5th International Conference on the Internet of Things (IOT), pp. 155-162. IEEE, 2015.

[17] Hong, Kirak, David Lillethun, Umakishore Ramachandran, Beate Ottenwälder, and Boris Koldehofe. "Mobile fog: A programming model for large-scale applications on the internet of things." In Proceedings of the second ACM SIGCOMM workshop on Mobile cloud computing, pp. 15-20. 2013.

[18] Bonomi, Flavio, Rodolfo Milito, Jiang Zhu, and Sateesh Addepalli. "Fog computing and its role in the internet of things." In Proceedings of the first edition of the MCC workshop on Mobile cloud computing, pp. 13-16. 2012.

ISSN: 2582-4104 